\title{
Integrating Topographic Measures to Explore the Protective Effects of Peonidin Against the N-Methyl-N-Nitrosourea Induced Photoreceptor Degeneration
}

\author{
Ye Tao a,c Tao Chen ${ }^{b}$ Zhongyu Liu ${ }^{d}$ Weiwei Xua Limin Qin ${ }^{a}$ Guanghua Peng ${ }^{a}$ \\ Yi Fei Huang ${ }^{\text {a }}$
}

aDepartment of Ophthalmology, General Hospital of Chinese PLA, Ophthalmology \& Visual Science Key Lab of PLA, Beijing, 'Department of Clinical Aerospace Medicine, Fourth Military Medical University, Xi'an, 'Department of Ophthalmology, Beidaihe Hospital of Chinese PLA, Beidaihe, ${ }^{\mathrm{d} D e p a r t m e n t}$ of gynaecology and obstetrics, General Hospital of Chinese PLA, Beijing, PR China

\section{Key Words}

Retinitis pigmentosa $\cdot \mathrm{N}$-Methyl $-\mathrm{N}$-nitrosourea $\cdot$ Topography $\bullet$ Peonidin

\begin{abstract}
Background/Aims: The pathphysiological properties of $\mathrm{N}$-Methyl $-\mathrm{N}$-nitrosourea (MNU) induced photoreceptor degeneration are similar to the hereditary retinitis pigmentosa (RP). The present study sought to explore the beneficial effects of the peonidin, a common aglycone form of anthocyanin, on the MNU induced photoreceptor degeneration via topographic measurements. Methods: The MNU administrated mouse received peonidin or vehicle injections, and then they were examined by electroretinography (ERG), multi electrode array (MEA), histological and immunohistochemistry studies. Results: The protective effects of peonidin on the MNU administrated retinas were systematically verified and quantified by topographic measures. The peonidin treatment could protect the photoreceptor against the MNU toxicity both functionally and morphologicaly. The most sensitive zone to peonidin therapy was sorted out, indicating that different rescuing kinetics existed between the retinal hemispheres and retinal quadrants. Moreover, the hyperactive spontaneous firing response and the debilitated light induced response in MNU administrated retinas could be partially reversed by peonidin treatment. To our knowledge, this was the first study to explore the pharmacological effects of peonidin on the electrophysiological properties of inner visual signal pathways. Conclusion: The peonidin could ameliorate the MNU induced photoreceptors degeneration and rectify the abnormities in the inner visual signal pathways. Future refinements of the knowledge cast insights into the discovery of a novel treatment for human RP.

Y. Tao, T. Chen and Z. Liu contributed equally to this publication and should be considered as co-first authors.

Professor Yi Fei Huang

and Professor Guanghua Peng

KARGER
Department of Ophthalmology, Chinese PLA General Hospital, 28 Fuxing Road, Haidian District, Beijing 100853, (China)

Tel.86-10-66937943, E-Mail huangyf301@163.com and E-Mail peng63088@163.com 


\section{Cellular Physiology Cell Physiol Biochem 2016;38:893-908 \\ \begin{tabular}{ll|l} 
and Biochemistry Published online: February 25, 2016 & $\begin{array}{l}\text { (c) } 2016 \text { The Author(s). Published by S. Karger AG, Basel } \\
\text { www.karger.com/cpb }\end{array}$ \\
\hline
\end{tabular} \\ Tao et al.: Retina, Neurotoxicity}

\section{Introduction}

Retinitis pigmentosa (RP) is an inherited neurodegenerative entity that characterized by the primary loss of photoreceptors, the progressive deterioration of visual fields, and the disorders of the visual signal pathways $[1,2]$. Currently, the pathologic mechanism of RP is not fully understood, neither no satisfactory treatment exists as more than 160 different mutated genes encoding proteins with remarkably diverse functions could cause RP [3]. The establishment of RP animal models is crucial for a better understanding of the disease progression, the underlying pathological mechanisms, and the development of therapeutic strategies. MNU (N-Methyl-N-nitrosourea) has been proven to be a validate toxicant for the selective induction of photoreceptor degeneration in mammalian retinas $[4,5]$. The mechanism leading to photoreceptor death is the principal alkylation of DNA, dependent on the action of alkyladenine DNA glycosylase (Aag), an enzyme that removes alkylated bases via cleavage of the glycosyl bond connecting the base to the sugar phosphate backbone, thus generating abasic sites that can be further processed by the base excision repair machinery. The MNU could induce $7 \mathrm{MeG}$ and 3MeA DNA lesions, both of which are Aag substrates. Photoreceptors would die when the repair process can no longer operate efficiently enough [6]. The electrophysiological and morphological properties of the MNU treated retinas are partially similar to that of the hereditary RP animal models and patients. Therefore, this highly reproducible chemical induced RP model has been widely utilized in the investigations on human RP [7-9].

Recently, several studies have shown that the oxidative stress contributes to the pathogenesis of retinal degeneration in both the hereditary and the MNU induced RP animal models [10-12]. Oxidative stress excessively elevates the poly adp-ribose polymerase (PARP) activity and triggers off photoreceptor apoptosis via its interaction with the transcription factors such as nuclear factor-kB (NF-kB) and activator protein-1 (AP-1).Moreover, it has been verified that the MNU enhances the reactive oxidative species (ROS) mediated photoreceptor apoptosis via the up-regulation of bax protein, the down-modulation of bcl-2 protein, and the activation of caspase families in retinas $[5,9]$. Since the primary events and the final outcomes of the RP retinas are the selective apoptotic photoreceptors death involving oxidative stress, these commons could be utilized for the further development of therapeutic strategies. Thus far, a series of therapeutic trials aimed at rescuing the photoreceptor from the MNU induced apoptosis, including the neurotrophic factors [13], caspase inhibitors [14], calcium channel blockers [15], and various antioxidants [16, 17].

Anthocyanin is a natural antioxidant widely distributed in fruits, plants and vegetables. Epidemiological studies have found that this water-soluble flavonoid is beneficial for variety of pathologies, including cardiovascular diseases, cancer, diabetes mellitus, neurodegeneration, and inflammation $[18,19]$. Particularly in the eyes, anecdotal researchers found that anthocyanin has potency in improving the scotopia symptoms and the microcirculation of retina. It acts as a stimulator of the rhodopsin resynthesis and plays an important role in the visual signal transduction [20,21]. Recently, the anthocyanin has been recognized as a retinal protective compound because it suppresses the light-induced photoreceptor damage by scavenging the ROS both in vivo and in vitro $[22,23]$. Anthocyanin also exert neuroprotective effects on the retinal ganglion cells (RGCs) and RPE cells via the anti-oxidation mechanism $[24,25]$. Furthermore, anthocyanin can inhibit AP-1 activation, an important mediator for photoreceptor apoptosis, by preventing the p38 from phosphorylation [26]. Therefore, we hypothesize that anthocyanin could suppress the MNU induced photoreceptors apoptosis, via or at least partly via the anti-oxidation mechanism. Peonidin is one of the most common aglycone forms of anthocyanin which are known as anthocyanidins [27]. The present study sought to clarify the protective effects of peonidin against the MNU-induced retinal damage by topographic measurements. Moreover, with the helping of the MEA recording, the formerly described MNU induced abnormalities of the visual signal transmission were systematically examined after peonidin treatment [28]. Our results suggested that peonidin could counteract the MNU induced photoreceptors degeneration and rectify the abnormities 


\section{Cellular Physiology Cell Physiol Biochem 2016;38:893-908 \begin{tabular}{ll|l} 
DOI: 10.1159/000443043 & $\begin{array}{l}\text { O 2016 The Author(s). Published by S. Karger AG, Basel } \\
\text { www.karger.com/cpb }\end{array}$ \\
\cline { 2 - 3 }
\end{tabular} \\ Tao et al.: Retina, Neurotoxicity}

in the inner visual signal pathways. Future refinements of the knowledge by the clinical study may identify peonidin as an effective treatment for human PR.

\section{Materials and Methods}

\section{MNU induced animal models and peonidin administration}

All experiments were conducted in accordance with the ARVO Statements for the Use of Animals in Ophthalmic and Vision Research. All efforts were made to minimize the number of animals used and their sufferings. All procedures regarding the use and the handling of the animals were conducted as approved by the Institutional Animal Care and Use Committee of the General Hospital of Chinese PLA. The C57/BL mice with both sexes used in this study were all 8-9 weeks old. All animals were maintained under standard laboratory conditions (room temperature $18^{\circ} \mathrm{C}$ to $23^{\circ} \mathrm{C}, 40 \%$ to $65 \%$ humidity, $12 \mathrm{~h}$ dark/light cycle) with food and water available ad libitum. The MNU (Sigma;St.Louis,MO) was kept at $-20^{\circ} \mathrm{C}$ in the dark. The MNU solution was dissolved in the physiologic saline containing $0.05 \%$ acetic acid just before use. MNUadministrated mouse received an intraperitoneal injection of MNU at the dose of $60 \mathrm{mg} / \mathrm{kg}$ body weight.

The peonidin used in the present study was purchased from Extrasynthese (Genay Cedex, France). Two hours after MNU administration, the mice of the experimental group received an intravenous injection of peonidin at the dose of $80 \mathrm{mg} / \mathrm{kg}$ body weight. Thereafter, they received an intravenous injection of peonidin once a day for consecutively four days. Meanwhile, the mice of the vehicle treated group received intravenous injections of physiological saline containing $0.05 \%$ acetic acid. Over all physiology and food intake of the experimental animals were monitored. No death occurred, and no clinical signs or system symptoms were evident in any of MNU administrated animals during the experiment. Experimental examinations were performed at P5 (day post MNU injection) and P14.

\section{ERG recording}

The ERG recordings were performed according to the previously described method [29]. Briefly, animals were weighed and dark-adapted overnight before recording and then were anesthetized by an intraperitoneal injection of ketamine $(80 \mathrm{mg} / \mathrm{kg})$ and chlorpromazine $(10 \mathrm{mg} / \mathrm{kg})$ under dim red light conditions. Animals were lightly secured to the stage in the UTAS Visual Diagnostic System with a Big Shot Ganzfeld (LKC Technologies, Gaithersburg, MD, USA). Platinum circellus record electrodes were placed on each cornea and a reference electrode placed subcutaneously between the eyes. White flashes with the intensity of $0.5 \log \mathrm{cd}-\mathrm{s} / \mathrm{m}^{2}$ were applied for stimulating the scotopic ERGs, and the inter-stimulus interval was $30 \mathrm{~s}$. Then mice were light adapted for $10 \mathrm{~min}$ at the intensity of $30 \mathrm{~cd}-\mathrm{s} / \mathrm{m}^{2}$. Photopic ERG measurements were recorded at the flash intensity of $1.48 \mathrm{log} \mathrm{cd}-\mathrm{s} / \mathrm{m}^{2}$ in the presence of continuous 30 Ganzfeld cd-s $/ \mathrm{m}^{2}$ background light, and the inter-stimulus interval was $0.4 \mathrm{~s}$. Totally 60 photopic responses and 10 scotopic responses were averaged for each measurement.

\section{MEA recording}

The MEA recording was described in detail in previous reports [28, 29]. Briefly, the animals were sacrificed under dim red light and their eyes were enucleated. The neural retina was gently removed from the pigment epithelium layer of the eye-cup and placed into the recording chamber. The electrode array used was composed of 64 electrodes, which were arranged in $8 \times 8$ layout (Alphamed Sciences, Osaka Japan). During recording, the ONH (optic nerve head) was fixed to the middle of the electrode array. These electrodes were classified into three groups according to their distances to the $\mathrm{ONH}$ : the central channels, the mid-peripheral channels, and the peripheral channels (Fig. 2A). Moreover, the global recording field was divided into four quadrants: superior temporal (ST), superior nasal (SN), inferior temporal (IT), and inferior nasal (IN). In order to obtain stable data, the retinal sample was placed in the recording chamber and perfused with oxygenated Ringer's solution $\left(95 \% \mathrm{O}_{2}\right.$ and $\left.5 \% \mathrm{CO}_{2}\right)$ for $1 \mathrm{~h}$ to adapt itself to the solution environment. The spontaneous activity of retina was recorded in dark environment for 10 minutes. The analog extracellular neuronal signals were detected by the MEA system (MED-64, Alpha med Sciences, Osaka, Japan) and were AC amplified, sampled at $20 \mathrm{kHz}$ and stored in a compatible computer for subsequent off-line software analysis(Neuroexplorer, Nex Technologies, MA, USA) software. The LEDs were driven by a computed stimulator to provide the retina a uniform full field illumination at a mean photonic 


\section{Cellular Physiology Cell Physiol Biochem 2016;38:893-908 \begin{tabular}{ll|l} 
DOI: 10.1159/000443043 & $\begin{array}{l}\text { O 2016 The Author(s). Published by S. Karger AG, Basel } \\
\text { www.karger.com/cpb }\end{array}$ \\
\cline { 2 - 3 }
\end{tabular} \\ Tao et al.: Retina, Neurotoxicity}

intensity of $850 \mathrm{mcd} \cdot \mathrm{sec} / \mathrm{m}^{2}$. A single retinal sample received a light stimulation protocol that lasted for 1 second. 10 retinal explants of the each group were used respectively at P5 and P14. Before spike detection, the field potentials were wiped off through a band pass filter (100 Hz to $3000 \mathrm{~Hz}$ ). These candidate spike waveforms were then sorted out by the Offline Sorter. The threshold for spike detection was set to four times the standard deviation (SD) of the mean vale of the measured signal for each electrode. These units without visual response were categorized as nonresponsive.

\section{Morphological evaluation by quantitative histology}

The eyes were enucleated and a hole was made in the nasal ora serrata with a needle for orientation purposes. The eyecups were immersed in a fixative solution $4 \%$ paraformaldehyde ((Dulbecco's PBS; Mediatech, Inc., Herndon, VA) for 6 hours. They were rinsed in PB (phosphate buffer), dehydrated in a graded ethanol series, and embedded in paraffin wax. Five sections (thickness: $4 \mu \mathrm{m}$ ) cut vertically through the ONH of each eye were stained with HE ( hematoxylin and eosin) and examined by light microscopy. ImagePro Plus software (Media Cybernetics, Silver Spring, MD) was used to outline the ONL (outer nuclear layer). The adjacent thickness of the outer nuclear layer was measured at $250 \mu \mathrm{m}$ intervals along the vertically superior-inferior axis by a single observer in a masked fashion. Averaged layer thicknesses at each point were calculated and plotted as a function of eccentricity from the $\mathrm{ONH}$, producing morph metric profiles across the vertical meridian. Similar to the MEA classification, the retina was divided into three regions: the central $(0-750 \mu \mathrm{m})$, the mid-peripheral $(750-1500 \mu \mathrm{m})$, and the peripheral $(1500-2250 \mu \mathrm{m})$ regions.

\section{Retinal flat mounts and immunohistochemistry}

The Retinal flat mounts were prepared according to previously described methods [30]. Briefly, the optic nerve bud and its surrounded sclera were cut from the back of the eyecup. Soft touching and gentle pressing by forceps on sclera of the eyecup to help separate the whole neuroretinal layer from the RPE layer, and the rest eyecup became the neuroretinal flat mounts. The flat-mounts were rained with PBS and were blocked in 2\% normal goat serum, 0.3\% Triton X-100 in 1\% BSA for 1 hour at room temperature, and then incubated overnight in the Peanut agglutinin conjugated to a Alexa Fluor 488 (1:200, L21409, Invitrogen) at $4{ }^{\circ} \mathrm{C}$ overnight. After extensive washing with buffer, the flat-mounts were incubated in Cy3-conjugated antirabbit IgG (1: 400, 711-165-152, Jackson ImmunoResearch Laboratories).The neuroretinal flat mounts were prepared by 4 cuts at 3, 6, 9 and 12 O'clock and were flattened under coverslips with anti-fade Vectashield mounting medium (Vector Laboratories, Burlingame, CA) for photographing. Fluorescence in flat mounts was analyzed with the Zeiss LSM 510 META microscope (Zeiss, thornwood, NY) fitted with Axiovision Rel. 4.6 software. The number of cones present within four $260 \times 260 \mu \mathrm{m}$ squares which located $1 \mathrm{~mm}$ superior, temporal, inferior, and nasal to the center of the optic nerve was determined. For quantification, all lighting parameters on the microscope and the camera software were standardized to ensure consistent stable lighting throughout the image capture procedure. A background image of a blank slide was taken for each sample set and was subtracted from the corresponding sample image. The retina was then selected and the integrated density (sum of the pixel values above threshold) of immune staining, as well as the total selected area and its mean labeling intensity (mean value of pixels above threshold) were measured.

\section{Statistical analysis}

In the MEA recording of RGCs activity, the clusters were first identified by a K-mean cluster algorithm, and then manually edited for clustering errors. The peristimulus time histograms (PSTHs) and the raster plots of individual units were used to categorize the ganglions. The ANOVA analysis followed by Bonferroni's post-hoc analysis was performed to examine the statistical differences between the normal control, the vehicle treated and the MNU treated animals. $P<0.05$ was considered significant. The values are presented as mean \pm standard error of the mean (S.E.M.) unless otherwise specified.

\section{Results}

The effects of the peonidin on the ERG of MNU administrated retinas

The ERG examination suggested that the photopic and scotopic ERGs of the MNU treated mouse were significantly impaired at the time point of P5 (Fig. 1A): the amplitude of the photopic b-wave in the vehicle treated mouse reduced by $63.8 \%$, and the scotopic 


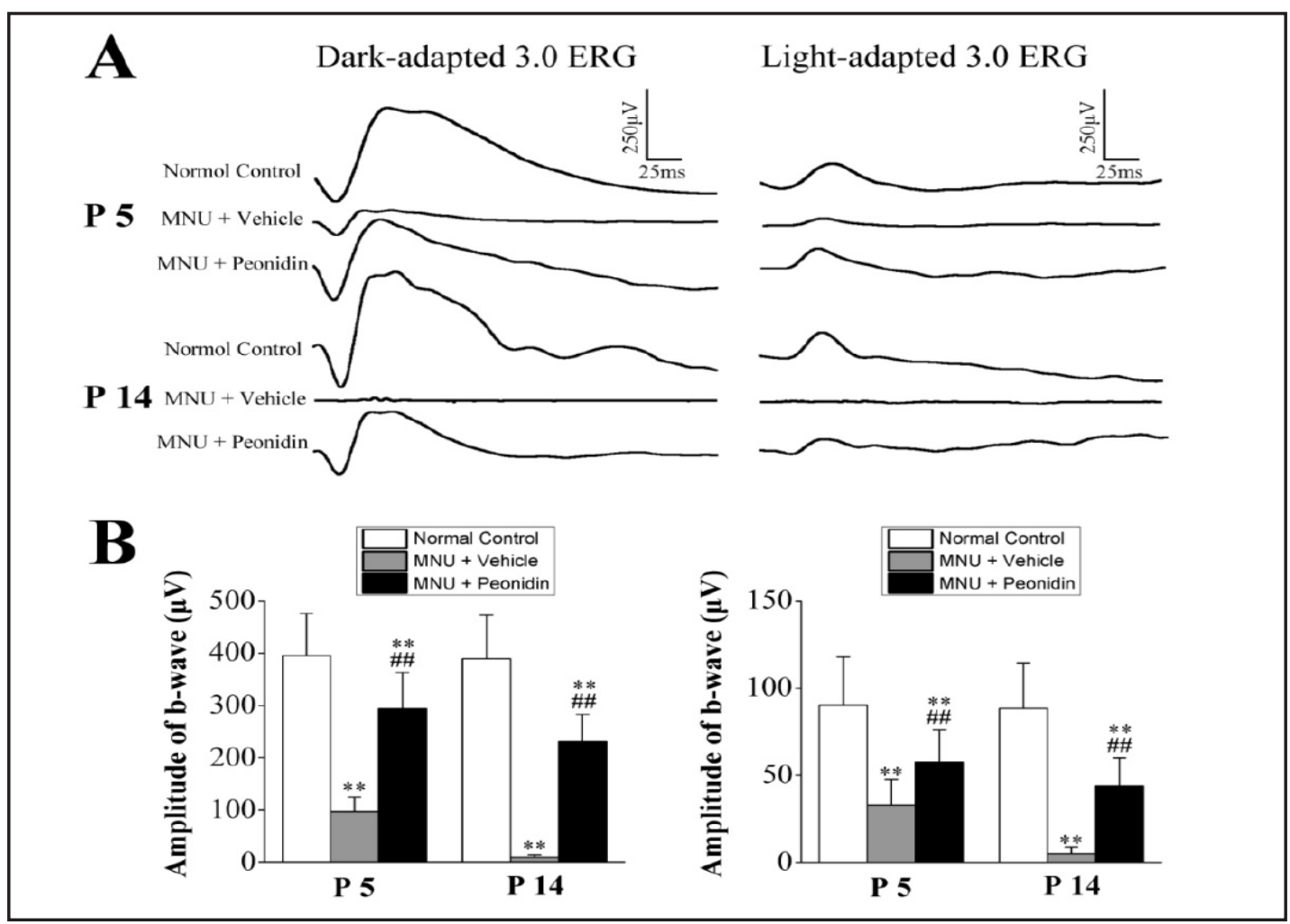

Fig. 1. (A) The representative ERG waveforms of the normal control, vehicle treated, and peonidin treated group at P5 and P14. (B) At P5, The amplitudes of both the photopic and scotopic b-waves of the peonidin treated mice were significantly larger than that of the vehicle administrated controls. Subsequently at P14, although the amplitudes of the photopic and scotopic b- waves of the peonidin treated mice significantly decreased compared with that at P5, they were significantly larger than that of the vehicle treated mice. (ANOVA analysis followed by post-hoc test; ${ }^{* *} P<0.01$ for differences compared with normal controls; ${ }^{\# \#} P<$ 0.01 for differences compared with vehicle treated; each group included 10 animals per examinational time point; All values represent Mean \pm S.E.M).

b-wave reduced by $76.3 \%$ in comparison with the normal controls; while the amplitude of the photopic b-wave in the peonidin treated mouse reduced by $35.2 \%$, and the scotopic b-wave reduced by $27.1 \%$ in comparison with the normal controls. The amplitudes of both the photopic and scotopic $b$-waves of the peonidin treated mouse were significantly larger than the vehicle treated controls (Photopic: $P<0.05$, peonidin treated vs. vehicle treated; Scotopic: $P<0.01$, peonidin treated vs. vehicle treated, $\mathrm{n}=10$, Fig. $1 \mathrm{~B}$ ). Subsequently at P14, the deterioration of photopic and scotopic ERGs in the MNU treated mouse progressed with time: no detectable waveform could be found in the vehicle treated mouse. The amplitudes of both the photopic and scotopic b- waves in the peonidin treated mouse also decreased significantly than that at P5 (Photopic: $P<0.01$, P14 vs. P5; Scotopic: $P<0.05$,P14 vs. P5, $\mathrm{n}=10$ ). However, the photopic and scotopic $\mathrm{b}$ - waves in the peonidin treated mouse were relatively preserved in comparison with the vehicle administrated mouse (Photopic: $P<$ 0.01 , peonidin treated vs. vehicle treated; Scotopic: $P<0.01$, peonidin treated vs. vehicle treated, $\mathrm{n}=10$ ).Moreover, the scotopic $\mathrm{b}$ - wave was more preferentially protected than the photopic b- wave in the peonidin treated mouse: $59.9 \%$ of the scotopic b-wave amplitude was retained in comparison with $50.1 \%$ of the photopic b-wave.

The topographic effects of the peonidin on the field potential of MNU administrated retinas

The light-induced field potentials were detected by the electrodes array, providing topographic information about global photoreceptor function. The field potentials of the 


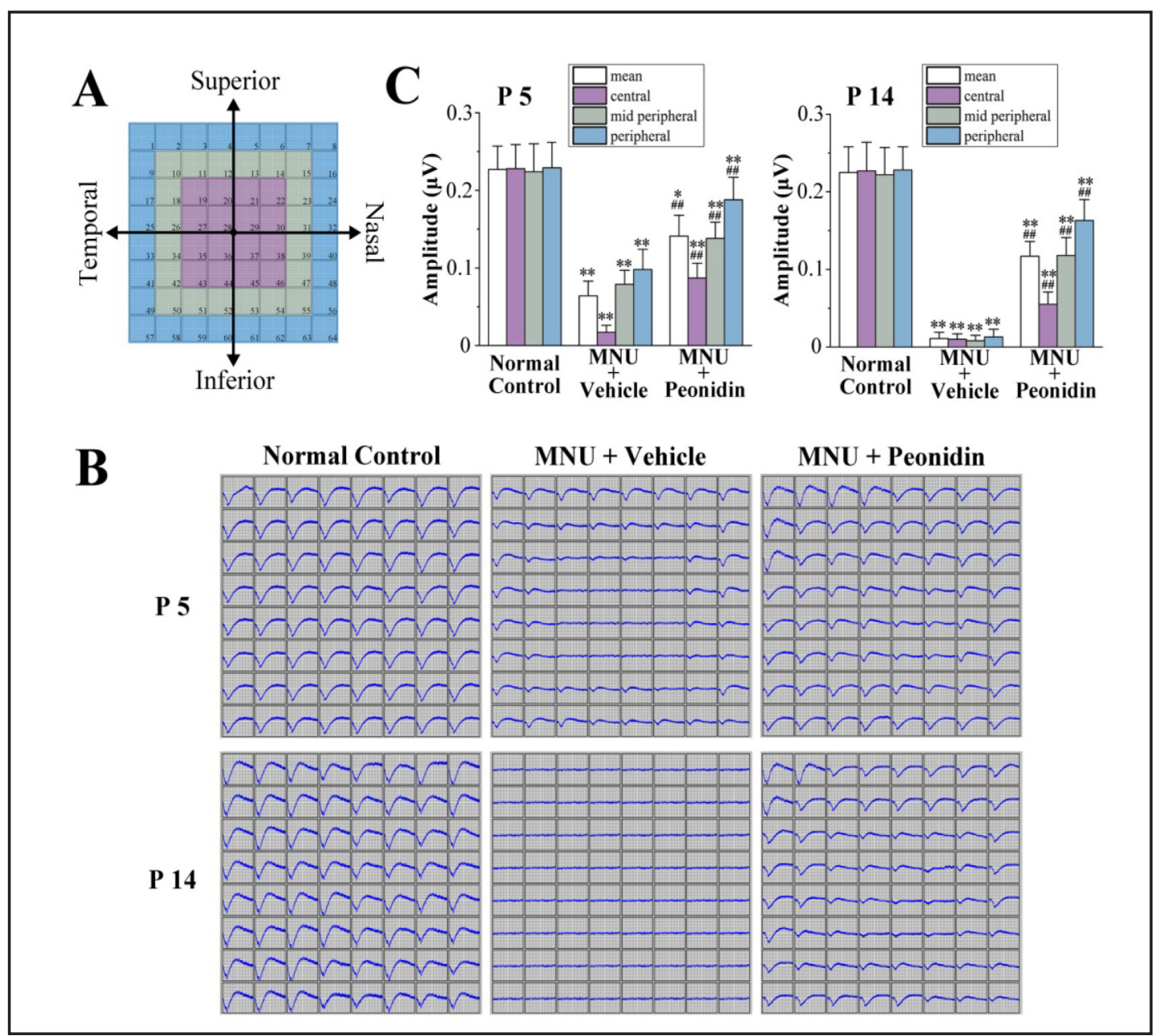

Fig. 2. (A) The MEA electrodes were classified into three groups according to their distances to the ONH: the central channels, the mid-peripheral channels, and the peripheral channels. Moreover, the global recording field was divided into four quadrants. (B) The field potentials of the vehicles treated retina showed positional degeneration: the negative waveforms of the field potential were terribly ruined and were almost undetectable in the central region; meanwhile, the field potential responses in the mid-peripheral region were less impaired; the amplitude of the peripheral region was significant larger than that of the mid-peripheral region. (C) In the peonidin treated mouse, the mean amplitude of field potential was significant greater than that of the vehicles controls. The amplitudes of all the three regions were larger than that of the vehicles controls. (ANOVA analysis followed by post-hoc test, $\mathrm{n}=10$; ${ }^{*} P<0.05$, ${ }^{* *} P<0.01$ for differences compared with normal controls; ${ }^{\#} P<0.01$ for differences compared with vehicle treated; each group included 10 animals per examinational time point; All values represent Mean \pm S.E.M).

vehicles treated retina showed positional degeneration at P5 (Fig. 2B): the negative waveforms of the field potential were terribly ruined and were almost undetectable in the central region (mean amplitude: $P<0.01$, vehicles treated vs. normal control, $\mathrm{n}=10$ ); the field potential responses in the mid-peripheral region were less impaired $(P<0.01$,mid-peripheral vs. central, $\mathrm{n}=10$ ); the amplitude of the peripheral region was significant larger than that of the mid- peripheral region $(P<0.01$, peripheral vs. mid-peripheral, $\mathrm{n}=10)$,especially the field potential waveforms in the peripheral ST quadrant were more consolidate than the other three quadrants. The mean amplitude in the peonidin treated mouse was significant larger than that in the vehicles controls $(P<0.01$, peonidin treated vs. vehicles treated, $\mathrm{n}=$ $10)$, although it was decreased by $39.3 \%$ in comparison with the normal controls $(P<0.01)$. In greater detail, the amplitudes of central, mid-peripheral, and peripheral regions in the 


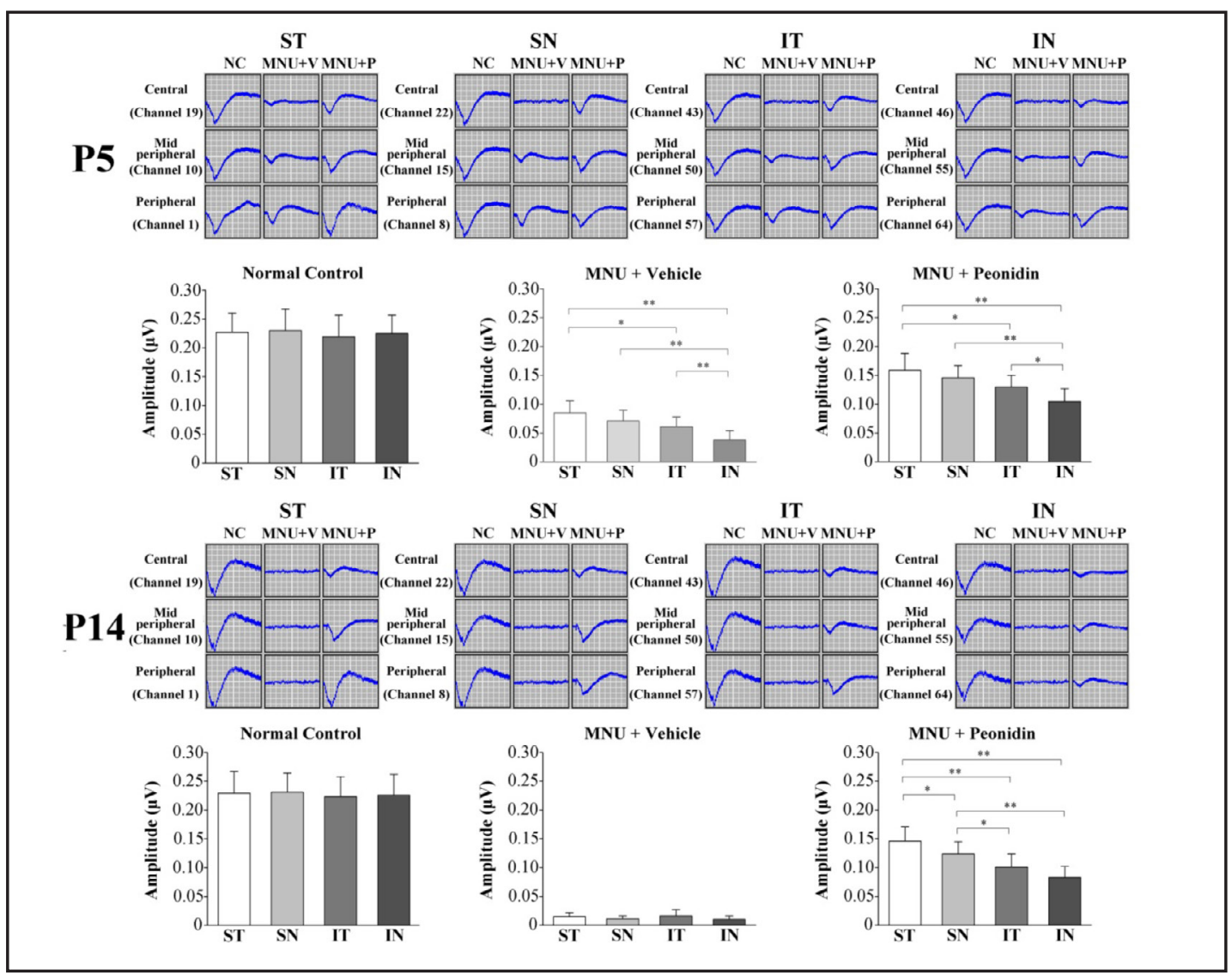

Fig. 3. Separately examined the field potentials of different quadrants in the peonidin treated mouse and it was found that the mean amplitude of the IN quadrant was the smallest one among the four quadrants, while the mean amplitude of the ST quadrant was the largest one at P5 and P14. The amplitudes of the field potentials in the peonidin treated mouse conformed to the following rule: ST $>$ SN $>$ IT $>$ IN. (ANOVA analysis followed by post-hoc test, $\mathrm{n}=10{ }^{*} P<0.05,{ }^{* *} P<0.01$ for differences between retinal quadrants; each group included 10 animals per examinational time point; All values represent Mean \pm S.E.M).

peonidin treated mouse were all significantly larger than the corresponding regions in the vehicles controls $(P<0.01$, peonidin treated vs. vehicles treated, $n=10$, Fig. $2 C)$.

By the time point of P14, no reliable waveforms of field potential was found in the vehicles treated mouse. On the contrary, a substantial proportion of the field potential was retained in the peonidin treated mouse. Moreover, the peripheral region seemed to be most efficiently preserved $(P<0.01$, peripheral vs. mid-peripheral, $\mathrm{n}=10$, Fig. $2 \mathrm{C})$. Separately examined the field potential of different quadrants in the peonidin treated mouse and it was found that the mean amplitude of the IN quadrant was the smallest one, while the mean amplitude of the ST quadrant was the largest one among the four quadrants (Fig. 3). The amplitude of the field potential in the peonidin treated mouse conformed to the following rule: $\mathrm{ST}>\mathrm{SN}>\mathrm{IT}>\mathrm{IN}$, indicating that photoreceptors with different positional characteristic showed comparative sensitivities to the peonidin protection.

The topographic effects of the peonidin on the morphology of MNU administrated retinas

In order to measure the topographic morphology of the retinas, sections were taken along the superior-inferior axis to access the vertical meridian of each hemisphere (Fig. 4A). At the time point of P5, the decrease of the ONL thickness was found in the vehicles treated mouse: the central region was subjected to terrible deterioration with almost vanished ONL, while the peripheral and mid-peripheral regions were relatively reserved. The retinal 


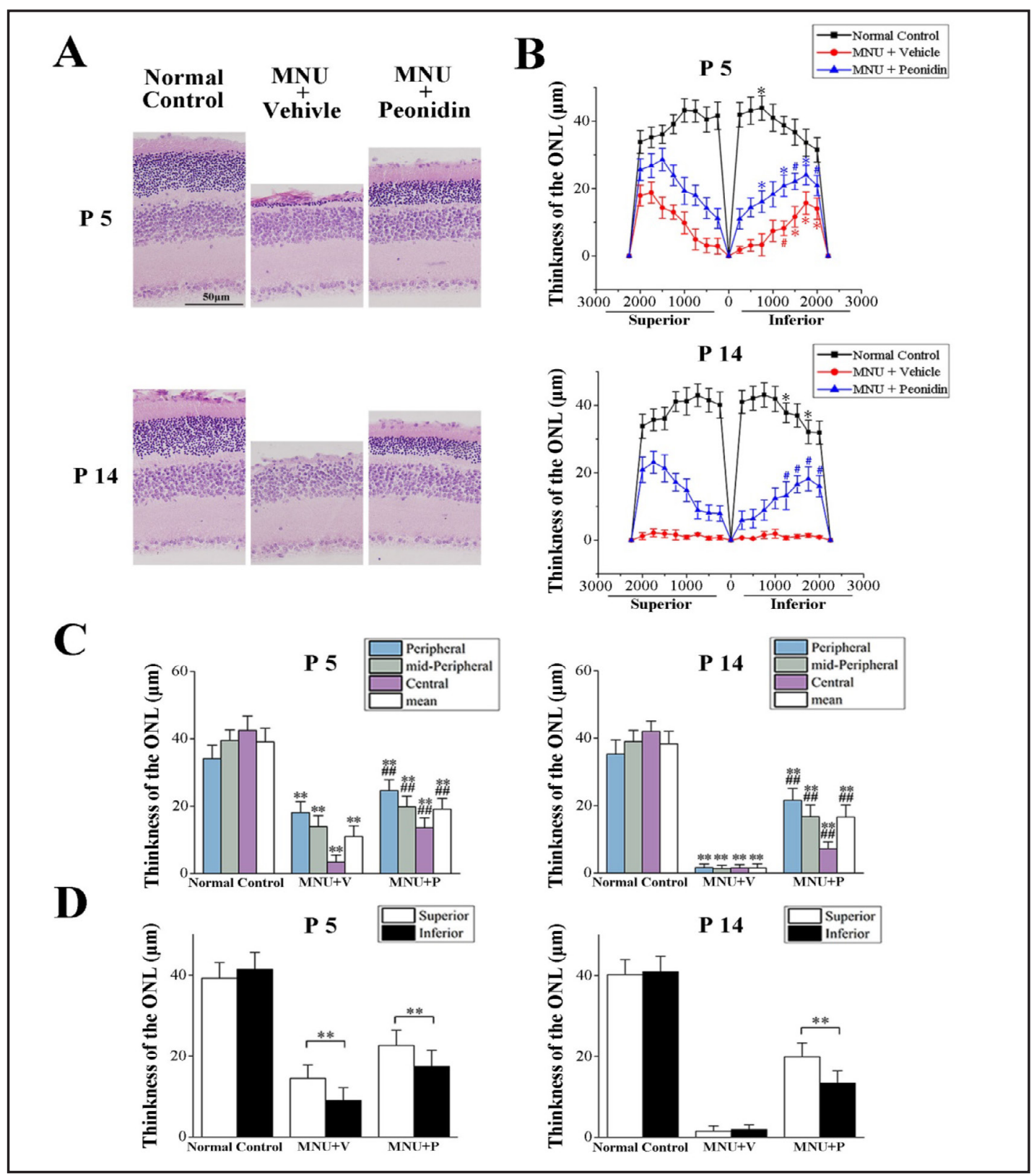

Fig. 4. (A) The decreased ONL thickness was found in the vehicles treated mouse, while the ONL of the peonidin treated mouse was effectively preserved. (B) Multiple points along the superior-inferior axis of the retina were measured. It was found that the peonidin induced protection ubiquitously distributed along the vertical meridian. In the peonidin treated mice, the ONL thickness of multiple points (especially peripheral region) were significantly larger than the corresponding points in the inferior hemisphere. (C) The retinal morphology of the peonidin treated mouse was less impaired and the ONL thicknesses of the peripheral, mid-peripheral and central regions were all significantly larger than that of the vehicles treated mouse. The decrease of ONL thickness in the peonidin treated mouse also progressed with time. However, a substantial proportion of the ONL thickness was preferentially retained in the peripheral and mid-peripheral regions. (D) In the peonidin treated mouse, the mean ONL thickness of the superior hemisphere was significantly larger than that of the inferior hemisphere both at P5 and P14. (ANOVA analysis followed by post-hoc test; B: ${ }^{*} P<0.05$, " $P<0.01$ for differences compared with the corresponding point in the superior points in the superior hemisphere; C: ** $P<0.01$ for differences compared with normal controls; ${ }^{\# \# ~} P<0.01$ for differences compared with vehicle treated; $\mathrm{D}:{ }^{* *} P<0.01$, superior compared with inferior hemisphere; each group included 10 animals per examinational time point; All values represent Mean \pm S.E.M). 


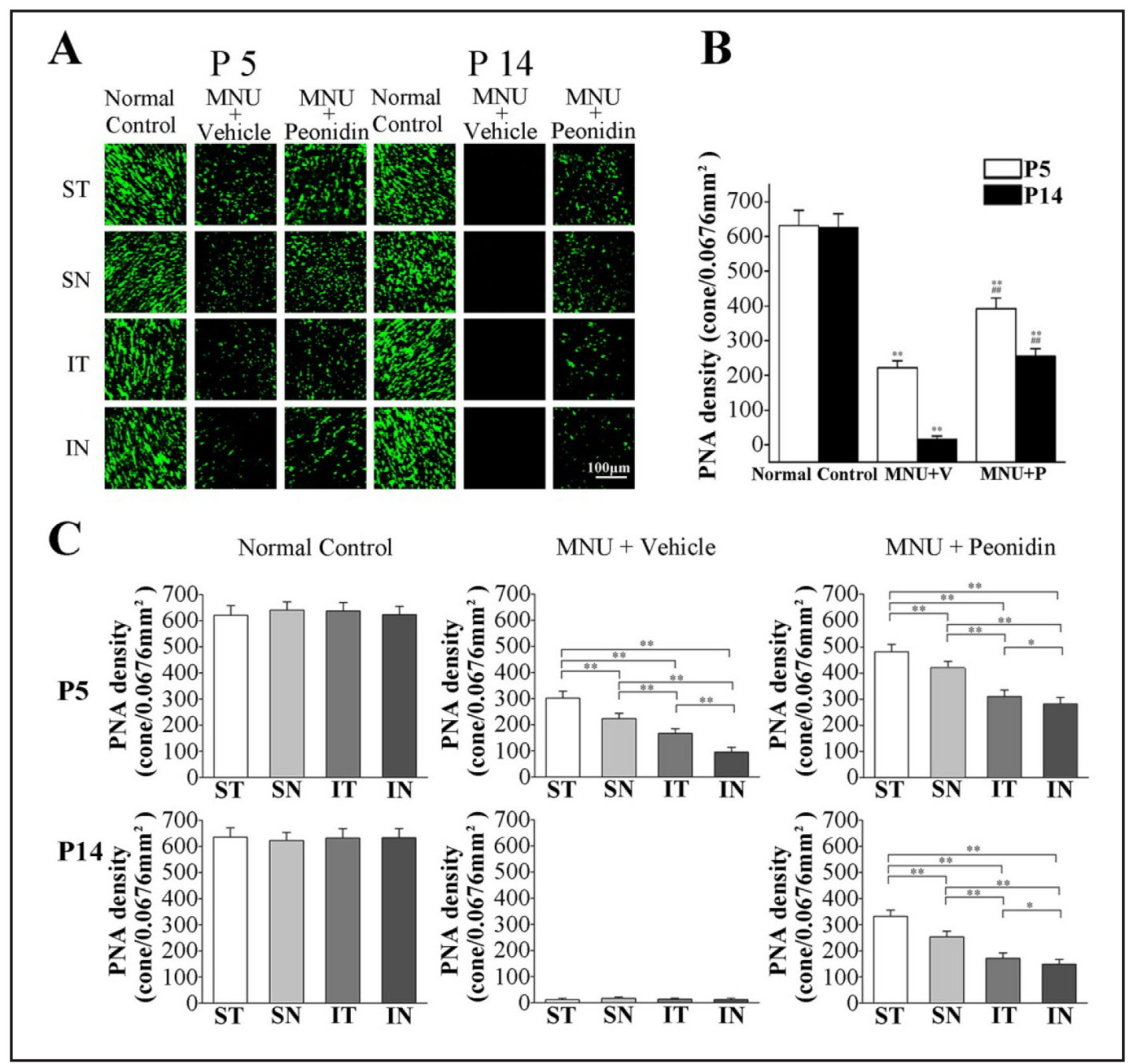

Fig. 5. (A) PNA immunostaining of different retinal quadrants. (B) The mean cone density substantially reduced in the vehicles treated mouse compared with that in the normal controls. While the mean cone density was significantly higher in the peonid treated mouse compared with that in the vehicles treated mouse. A larger drop of cone density was found in the vehicles treated mouse in comparison with the peonid treated mouse. (C) In the peonidin treated retinas, the most resistant cone was preferentially distributed in the ST quadrant: the cone density of this region was significantly higher than the other quadrants both at P5 and P14; while the cone density of the IN quadrant was always the smallest one. (ANOVA analysis followed by post-hoc test, $\mathrm{n}=10$; $\mathrm{B}$ : ${ }^{* *} P<0.01$ for differences compared with normal controls; ${ }^{\# \# ~} P<0.01$ for differences compared with vehicle treated; $\mathrm{C}$ : ${ }^{*} P<0.05,{ }^{* *} P<0.01$ for differences between retinal quadrants; each group included 10 animals per examinational time point; All values represent Mean \pm S.E.M).

morphology of the peonidin treated mouse was less impaired and the ONL thicknesses of the peripheral, mid-peripheral and central regions were all significantly larger than that of the vehicles treated mouse $(P<0.01$, peonidin treated vs. vehicle treated, $n=10$, Fig. 4B). At the time point of P14, the ONL of the vehicles treated mouse were absolutely ruined by the MNU administration, including the peripheral and mid-peripheral areas (Fig. 4C). In the peonidin treated mouse, the decrease of ONL thickness also progressed with time $(P<0.01$, P5 vs. P14, $\mathrm{n}=10$ ). However, the ONL in the peripheral and mid-peripheral regions were preferentially retained with larger thickness than the central region. Furthermore, we separately examined the ONL thickness of the superior and inferior retinas and found that the two hemispheres in the peonidin treated retinas showed different sensitivities to the peonidin treatment: the

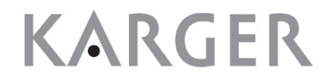




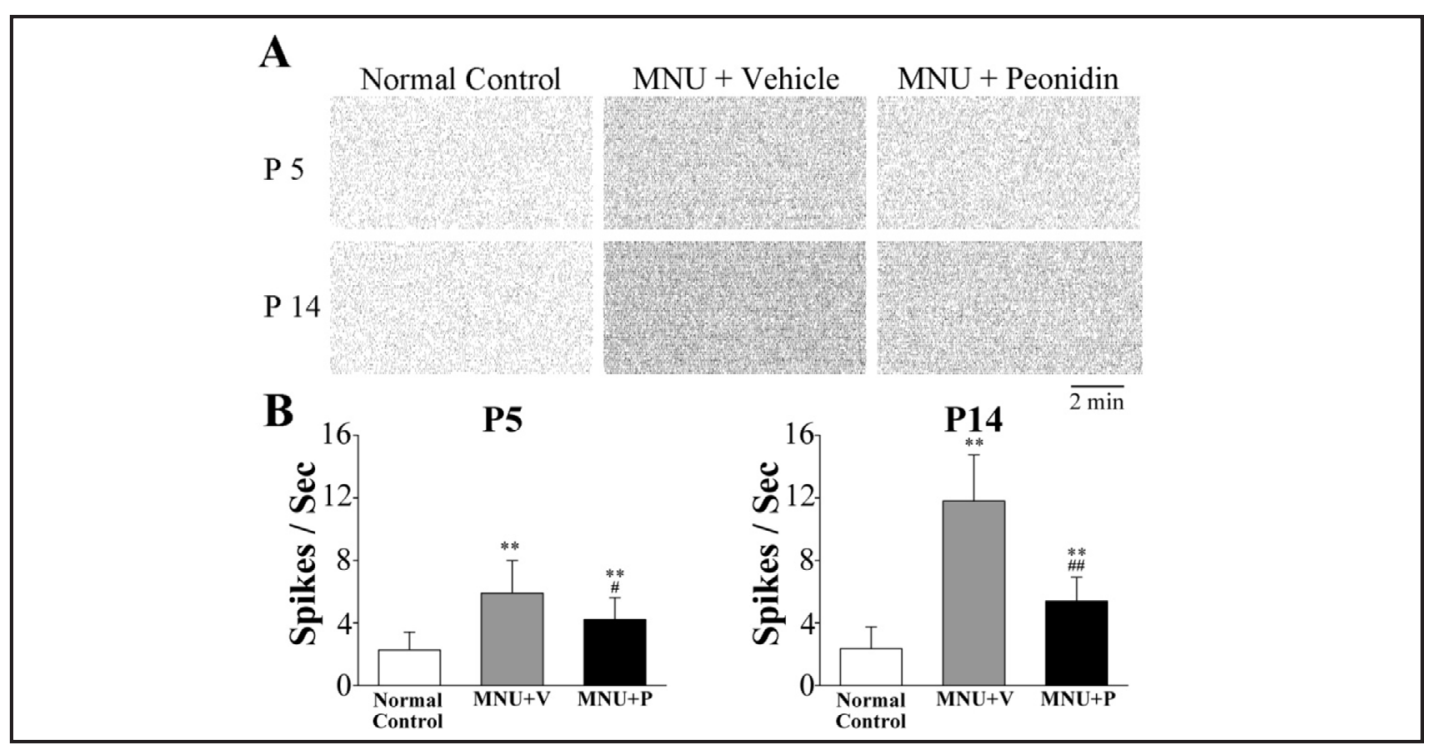

Fig. 6. (A) The raster plots of the firing spikes suggested a significant increase in the firing rate of the vehicle treated retina respectively at P5. The spontaneous firing rate of the peonidin treated retina also increased, but it was relatively lower than that of the vehicle treated controls. (B) At P14, the spontaneous hyperactivity of the vehicle treated retinas progressed with time, although the photoreceptors activity has been absolutely destroyed by MNU. In contrast, the spontaneous firing rate of the peonidin treated retinas remained stable. (ANOVA analysis followed by post-hoc test, $\mathrm{n}=10$; ${ }^{* *} P<0.01$ for differences compared with normal controls; " $P<0.05$, \#\# $P<0.01$ for differences compared with vehicle treated; each group included 10 animals per examinational time point; All values represent Mean \pm S.E.M).

ONL thickness of the superior hemisphere in the peonidin treated mouse was significantly larger than that of the inferior hemisphere at P5 and P14 $(P<0.01$, superior vs. inferior, $\mathrm{n}=$ 10, Fig. 4D).

The cones of the MNU administrated retinas were rescued by the peonidin

As the rods account for at least $96 \%$ of the total photoreceptors in the mouse retina, the ONL thickness mainly reveals the rod integrity and should be considered as an indicator of the rod number and vitality. However, whether the minority cones were rescued by peonidin remained unknown. In order to verify the sensitivity of the cones to peonidin treatment, we examined the PNA immunostaining which specifically located in the outer segment of the cones (Fig. 5A). At P5, the cone density substantially reduced in the vehicles treated mouse. While the cone density in the peonidin treated mouse was significantly higher in comparison with the vehicles treated mouse $(P<0.01$ peonidin treated vs. vehicles treated, $\mathrm{n}=10$, Fig. 5B). Compared with the normal controls, a significant drop of cone density was found in the vehicles treated mouse (by $\sim 65.9 \%, P<0.01$, vehicles controls vs. normal controls, $\mathrm{n}=10$ ), while a lesser drop was found in the peonidin treated mouse (by $\sim 38.6 \%, P<0.01$, peonidin treated vs. normal control, $\mathrm{n}=10$ ). By P14, the cone immunostaining in the vehicles treated mouse were eliminated by the MNU administration. However, the cone density of the peonidin treated mouse was significantly higher $(\mathrm{P}<0.01$ peonidin treated vs. vehicles control, $\mathrm{n}=10$ ). By this time point, $40.7 \%$ of the cone immunostaining was preserved in the peonidin treated mouse. Moreover, the cone density of the ST quadrant was significantly higher than the other quadrants in the peonidin treated retinas at P5 and P14; while the cone density of the IN quadrant was always the smallest one (Fig. 5C).

The effects of peonidin on the visual signal transmission of MNU administrated retinas

The MEA system was used to monitor the firing spikes of the RGCs and to verify the protective effects of peonidin on the visual signal transmission of inner retinal circuits. A 


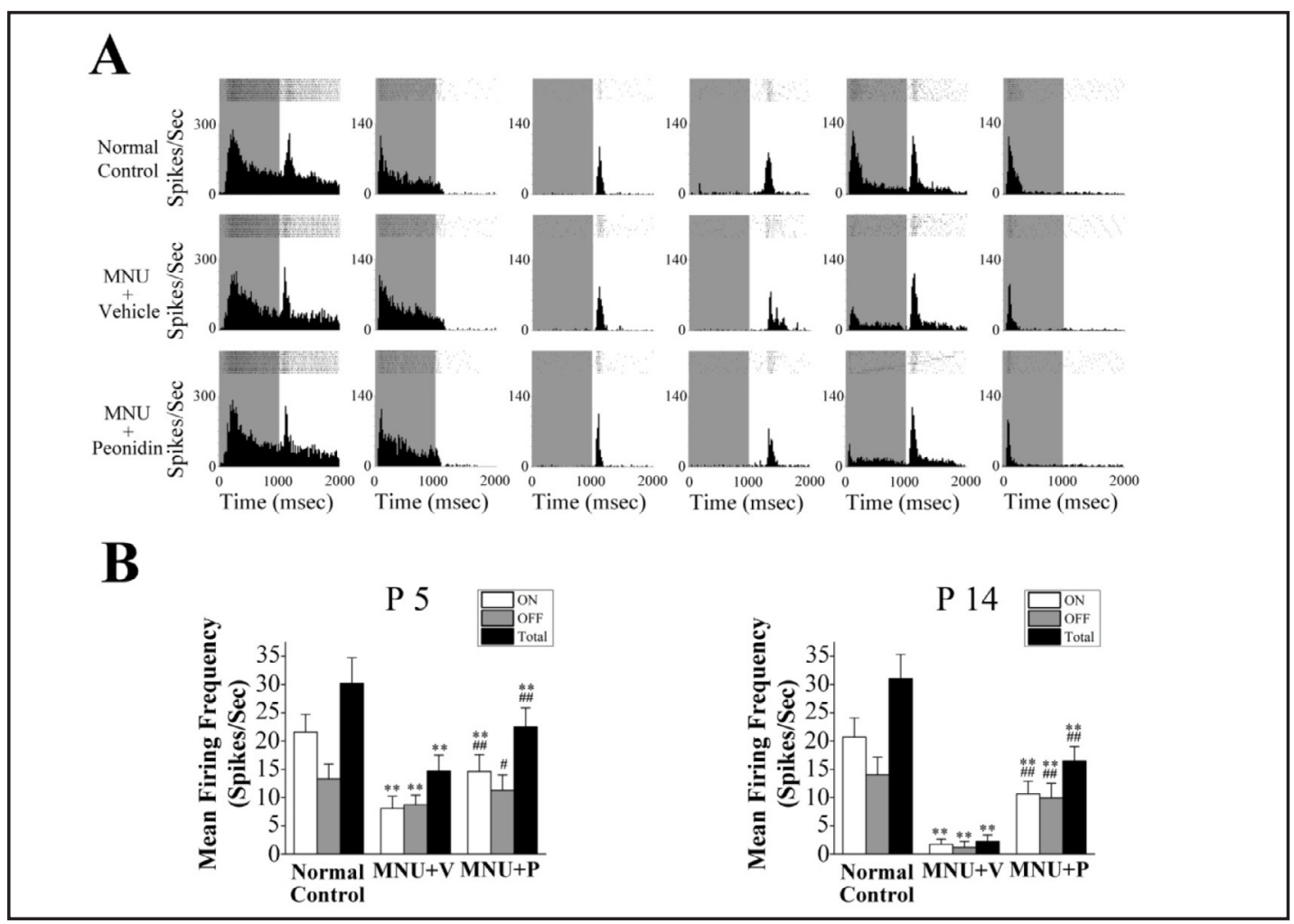

Fig. 7. (A) Six categories RGCs populations of each animal group were distinguished by their responsive characters to the light stimulus. Those are: responding predominantly to light onset (ON), to light offset (OFF), or to both (ON-OFF); sustained response to light onset (sustained ON); sustained response to light on and offset (sustained ON-OFF); sluggish response to light offset (delayed OFF). Peristimulus time histograms (PSTHs) under the raster plots also noted the existence of these RGCs populations in retinas. (B) At P5, the light induced firing rate of the vehicle treated mouse decreased significantly in comparison with normal controls. While the light induced response in the peonidin treated mouse was efficiently rescued. Although the firing rate of the $\mathrm{ON}$ response in the peonidin treated mouse decreased significantly at $\mathrm{P} 5$, the firing rate of the OFF response was not impaired. Subsequently at P14, both the firing rates of the ON and OFF response decreased significantly compared with that in the normal controls, but they are significantly higher than that in the vehicle treated mouse. (ANOVA analysis followed by post-hoc test, $\mathrm{n}=10$; ${ }^{* *} P<0.01$ for differences compared with normal controls; ${ }^{\#} P<0.05$, ${ }^{\# \# ~} P<0.01$ for differences compared with vehicle treated; each group included 10 animals per examinational time point; All values represent Mean \pm S.E.M).

significant increase of the spontaneous firing rate was found in the vehicle treated retina at P5 $(P<0.01$, vehicle treated vs. normal controls, $n=10$, Fig. $6 \mathrm{~A})$. The spontaneous firing rate of the peonidin treated retina also increased significantly $(P<0.01$, peonidin treated vs. normal control, $\mathrm{n}=10)$, but it was lower than that of the vehicle treated controls $(P<0.05$, peonidin treated vs. vehicle treated, $\mathrm{n}=10$ ). By P14, the spontaneous hyperactivity of RGCs in the vehicle treated retinas progressed with time $(P<0.01, P 14$ vs. $P 5, n=10)$, although the photoreceptors has been completely destroyed by MNU. In contrast, the spontaneous firing rate of the peonidin treated retinas remained stable $(P>0.05$, p14 vs. $P 5, n=10$, Fig. 6B).

Light induced RGCs responses were evoked by full-field stimulus and the firing spikes were extracted by the off-line Spike Sorter. Six categories of RGCs populations in each retinal group were distinguished by their responsive characters to light stimulus, indicating that the basic physiological response types and a substantial part of RGCs function were retained in the vehicle treated and peonidin treated retinas, despite the signal inputs from photoreceptors had been impaired by MNU (Fig. 7A). At P5, the light induced firing rate of the vehicle treated controls decreased significantly: the firing frequency of both the $\mathrm{ON}$ 
and OFF response attenuated in comparison with the normal controls $(P<0.01$, vehicle treated vs. normal control, $n=10$ ). This degeneration progressed till P14 as no reliable light induced response could be detected. However, the light induced response in the peonidin treated mouse was efficiently rescued: the firing rate was nearly two folds to that of the vehicle treated. Although the firing rate of the ON response in the peonidin treated mouse decreased significantly $(P<0.01$, peonidin treated vs. normal control, $\mathrm{n}=10)$, the firing rate of the OFF response was not impaired $(P>0.05$, peonidin treated vs. normal control, $\mathrm{n}$ $=10$ ). Subsequently at P14, both the firing rates of the ON and OFF response substantially decreased compared with that of normal controls $(P<0.01$, peonidin treated vs. normal control, $\mathrm{n}=10)$, however, they are significantly higher than that of the vehicle treated $(P<$ 0.01 , peonidin treated vs. vehicle treated, $n=10$, Fig. 7B).

\section{Discussion}

The establishment of animal models resembling human RP is crucial for a better understanding of the pathologic mechanisms and for further development of therapeutic strategies. The MNU induced selective photoreceptor apoptosis in mammalian eyes which partially mimics RP pathology and this highly reproductive model have been widely utilized in the pathologic and pharmacological investigations[7-9]. It has been found that oxidative stress plays a significant role in the photoreceptor degeneration of the MNU induced or hereditary RP model: abundant reactive oxidative species (ROS) cause mitochondria membrane depolarization and has been ascribed a central role in the apoptotic cascades. It was shown that ROS and calpain could significantly contribute to the caspase- independent apoptotic pathway in the RP retinas [31,32]. Moreover, a novel study has found that the peroxynitrite generated from ROS and nitric oxide (NO) exacerbates the oxidative damage and contributes to the photoreceptor death in RP [33]. Therefore, the surplus ROS in the $\mathrm{RP}$ retinas should be neutralized by antioxidant defense system or exogenous antioxidants, otherwise they will interact with functional macromolecules such as the unsaturated lipids, proteins, deoxyribonucleic acids, which are all critical for photoreceptor survival.

Peonidin is a potent antioxidant which is capable of counteracting the oxidative damages, scavenging the surplus oxidative species (ROS), and rectifying the abnormities in the apoptotic cascade[18, 19]. Taken together with its ability to attenuate inflammation which also contributes to the etiology of RP, it is reasonable to hypothesize that peonidin might act as novel therapeutic elements to arrest the photoreceptor degeneration in RP retinas [23]. In the present study, the protective effects of peonidin on the global retina of MNU administrated mouse were systematically assessed and quantified. Thereby, the most sensitive zone to peonidin therapy was sorted out by topographic measures, indicating different rescuing kinetics existed between the retinal hemispheres and retinal quadrants. More importantly, to our knowledge, this was the first study to explore the pharmacological effects of peonidin treatment on the electrophysiological properties of inner visual signal pathways.

Herein, it was shown that the intravenous administration of peonidin could ameliorate the MNU induced photoreceptor degeneration both morphologically and functionally till the end of the follow-up (14 days post MNU administration). Our MEA data suggested that positional photoreceptors in the MNU treated retinas showed distinctive sensitivities to the peonidin treatment: the peripheral photoreceptors, especially these in the ST quadrant were most efficiently preserved; On the contrary, the peonidin administration was futile to save these central photoreceptors. Agreed well with the MEA results, the ONL microstructure of peripheral region in the superior hemisphere was the most consolidated zone after peonidin treatment. Intriguingly, immunostaining of the whole mounts also suggested that the cones, especially these distributed in the ST quadrant were most efficiently rescued by the peonidin administration. The ERG examination and the firing spike analysis provide functional information about the photoreceptors and the downstream visual signal pathways: both the 
photopic and scotopic ERG were effectively preserved in these peonidin treated retinas, and the abnormalities caused by the MNU neurotoxicity, such as the spontaneous hyperactivity of RGCs, could be partially reversed by peonidin treatment. A substantial proportion of the light induced signal of the RGCs was also recued by the peonidin administration: the basic $\mathrm{ON}$ and $\mathrm{OFF}$ visual signal pathway were efficiently retained for at least 14 days. As the ultimate grade of retinal neurons, the RGCs collect the visual signals form noisy presynaptic inputs and then convert them into the action potentials, which is considered as the standard phenotype of visual information that eventually conveyed to the occipital cortex [34-36]. The hyperactivity of spontaneous activity and the debilitating light induced response of the RGCs have been found in a variety of retinal degenerations, implying significant disturbances in the RGCs electrophysiological properties and the visual signal transmission pathways [3740]. The spontaneous RGCs hyperactivity is hazardous since it could add undesirable noise to the communication between the eye and the brain and eventually affect the authentic effectiveness of visual function [41]. Together with the decreased robustness of light induced RGCs Response, these alterations would result in the deteriorated signal-noise ratio (SNR) of the degenerative retinas [34, 42]. Therefore, the hyperactive spontaneous activity and the debilitating light induced response of the RGCs would impair the responsivity and disturb the reliability of visual transmission in the MNU administrated retinas [28]. Our MEA data suggests that peonidin exerts beneficial effects on both the spontaneous and the light induced response of RGCs, and thereby improve the signaling efficiency and fidelity of inner retinal visual pathways.

The MNU induced RP model has been utilized to develop potential therapeutic strategies for human RP. However, none of these therapeutic trials are based on the topographic characteristics of the MNU induced RP model. As the suitable experimental technologies able to synchronously monitor multi-positional photoreceptor characteristics are not always available, the positional photoreceptor vitality is systematically examined after treatments, On the other hand, it has been found that focal photoreceptors showed different resistance to the MNU toxicity and the induced photoreceptors dysfunction underwent a distinct spatial- and time-dependent progression $[5,28]$. Therefore, the topographic characteristics of the MNU induced photoreceptor degeneration are crucial, especially for these therapeutic explorations which seek to quantify the therapeutic efficiency in the MNU animal models: the anticipated protective effect could be directly affected by the retinal locations that selected for observation, and closely relate to the time when the evaluation is conducted. Without the supports of the topographic evaluation technologies, the temporarily normal zone would be readily mistaken for the effectively rescued zone. Consequently, the experimenter would be confronted with higher risk of positive errors if they selected asymmetric retinal locations between the control and the experimental groups for therapeutic effect analysis. The present study provides an example of integrating the topographic technologies into the evaluation of therapeutic effects on retinopathy, and the vitality of local photoreceptor was isolated. Admittedly, the greatest protection against photoreceptor death is seen in the far periphery retina does not support a major benefit for central vision, which is the most vital area to preserve for useful human vision. We proposed that this topographic characteristic might be considered as a disadvantage of the peonidin therapy. Whether the central vision of the RP patients could be efficiently rescued by peonidin therapy remained to be evaluated by future clinical cohorts. Furthermore, although the fate of the MNU injured photoreceptor resembles RP, the mechanistic underpinning and the kinetics are very different. Most forms of RP are caused by the mutations in rod- related genes which are crucial for retinal function, such as phototransduction, protein trafficking, phagocytosis.etc, leading to gradual demise of rods [1-3]. However, the MNU induced rapid photoreceptor death via the alkylation of DNA and the activation of downstream apoptotic cascade [6, 7]. Therefore, the novel and surprising observation that cones are also killed by MNU toxicity does not correspond to cone degeneration in RP either, since demise of this population occurs in a secondary wave in the RP retinas, long after rods have degenerated. These discrepancies are crucial in any study which proposes the clinical testing of candidate molecules. 


\section{Cellular Physiology Cell Physiol Biochem 2016;38:893-908 \begin{tabular}{ll|l} 
DOI: 10.1159/000443043 & $\begin{array}{l}\text { O 2016 The Author(s). Published by S. Karger AG, Basel } \\
\text { www.karger.com/cpb }\end{array}$ \\
\cline { 2 - 3 }
\end{tabular} \\ Tao et al.: Retina, Neurotoxicity}

The bioavailability of the peonidin to retina is another pharmacological issue yet to be addressed. Effective delivery of the peonidin to the targeted retinal tissue is a formidable task. Repeated intraocular or intravitreal injections are problematic for RP mouse or patients due to the delicacy of eye structures and the propensity for cataract formation. On the other hand, pharmacokinetic data indicates that the absorption of anthocyanin is relatively poor in human: less than $1 \%$ after oral administration was absorbed [43-45]. The concentration of peonidin in plasma after oral supplements is far below the level required to exhibit cytoprotective effects in vitro. However, after absorbed into the plasma, the peonidin can readily cross the mammalian blood-retinal barrier and distribute in ocular tissues as intact forms $[46,47]$. Therefore, the intravenous administration in the present study may act as a promising delivery method because it could enhance the blood level of peonidin.

We are not that optimistic a single agent such as peonidin is potent enough to produce ideal therapeutic effects on the global retina. It is likely that benefits by reducing oxidative damage to photoreceptors and simultaneously increasing the threshold for apoptosis (eg. neurotrophic treatment) can be synergistic $[48,49]$. Peonidin can also be utilized as supplements forgene therapy until the oxygen status of RP retina recovers to normal. Moreover, Pharmacological safety issues need to be addressed before further drug development. As the slow progression nature of RP requires long-term administration, peonidin should be present at the right time, correct cellular compartment, and appropriate concentration to RP patients. Additionally, possible toxicity of chronic peonidin supplementations should be efficiently evaluated.

Gene therapy for RP remains to be challenging due to the tremendous genetic heterogeneity of this disease. Targeting a patho-physiological process which is common to all the mutation phenotypes (e.g. apoptosis, oxidative damages) could serve as a more promising and general alternative for RP treatment. Peonidin is a potential drug for RP due to its anti-apoptosis and anti oxidation properties. Our results indicate that the peonidin could counteract the MNU induced photoreceptors degeneration and rectify the abnormities in the inner visual signal pathways. These topographic effects of peonidin on the MNU induced photoreceptor degeneration would enrich our pharmacological knowledge about this natural antioxidant. It is our hope that the protective effects as evidenced by the present study could cast insight into the discovery of a pervasive treatment for human RP.

\section{Acknowledgements}

This work is supported by the the National Key Basic Research Program of China (973 Program: No.2013CB967001).

\section{Disclosure Statement}

The authors declare that there is no conflict of interests.

\section{References}

1 Hartong DT, Berson EL, Dryja TP: Retinitis pigmentosa. Lancet 2006;368:1795-1809.

2 Shintani K, Shechtman DL, Gurwood AS: Review and update: current treatment trends for patients with retinitis pigmentosa. Optometry 2009;80:384-401.

3 Baumgartner WA: Etiology, pathogenesis, and experimental treatment of retinitis pigmentosa. Med Hypotheses 2000;54:814-824.

4 Tsubura A, La YC, Miki H, Sasaki T, Uehara N, Yuri T, Yoshizawa K: Review: animal models of N- methyl -N - nitrosourea -induced mammary cancer and retinal degeneration with special emphasis on therapeutic trials. In Vivo 2011;25:11-22. 


\section{Cellular Physiology Cell Physiol Biochem 2016;38:893-908

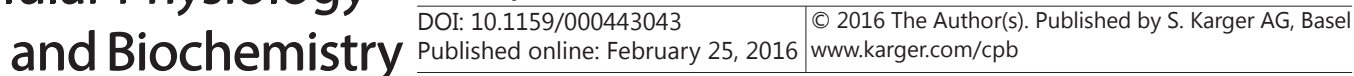 \\ Tao et al.: Retina, Neurotoxicity}

5 Tsubura A, Yoshizawa K, Kuwata M, Uehara N: Animal models for retinitis pigmentosa induced by MNU: disease progression, mechanisms and therapeutic trials. Histol Histopathol 2010;25:933-944.

6 Lisiane B. Meira, Catherine A. Moroski-Erkul, Stephanie L. Green, Jennifer A. Calvo, Roderick T. Bronson, Dharini Shah, and Leona D. Samson: Aag-initiated base excision repair drives alkylation-induced retinal degeneration in mice. Proc Natl Acad Sci USA 2009;106:888-893.

7 Yoshizawa K, Nambu H, Yang J, Oishi Y, Senzaki H, Shikata N, Miki H, Tsubura A: Mechanisms of photoreceptor cell apoptosis induced by N-methyl-N-nitrosourea in Sprague-Dawley rats. Lab. Invest 1999;79:1359-1367.

8 Komeima K, Rogers BS, Lu L, Campochiaro PA: Antioxidants reduce cone cell death in a model of retinitis pigmentosa. Proc Natl Acad Sci USA 2006;103:11300-11305.

9 Gao Y, Deng XG, Sun QN, Zhong ZQ: Ganoderma spore lipid inhibits N-methyl -N -nitrosourea-induced retinal photoreceptor apoptosis in vivo. Exp Eye Res 2010;90:397-404.

10 Yu DY, Cringle S, Valter K, Walsh N, Lee D, Stone J: Photoreceptor death, trophic factor expression, retinaloxygen status, and photoreceptor function in the P23H rat. Invest Ophthalmol Vis Sci 2004;45:20132019.

11 Shen J, Yang X, Dong A, Petters RM, Peng YW, Wong F, Campochiaro PA: Oxidative damage is a potential cause of cone cell death in retinitis pigmentosa. J Cell Physiol 2005;203:457-464.

12 Curtin JF, Donovan M, Cotter TG: Regulation and measurement of oxidative stress in apoptosis. J Immunol Methods 2002;265:49-72.

13 Xu H, Yang J.N, Li X.K, Zheng Q Zhao W, Su ZJ, Huang YD: Retina protective effect of acidic fibroblast growth factor after canceling its mitogenic activity. J. Ocul Pharmacol Ther 2008;24:445-451.

14 Petrin D, Baker A, Coupland SG, Liston P, Narang M, Damji K, Leonard B, Chiodo VA, Timmers A, Hauswirth W, Korneluk RG, Tsilfidis C: Structural and functional protection of photoreceptors from MNU-induced retinal degeneration by the X-linked inhibitor of apoptosis. Invest Ophthalmol Vis Sci 2003;44:2757-2763.

15 Oka T, Nakajima T, Tamada Y, Shearer T.R, Azuma M: Contribution of calpains to photoreceptor cell death in N-methyl-Nnitrosourea-treated rats. Exp Neurol 2007;204:39-48.

16 Emoto Y, Yoshizawa K, Uehara N, Kinoshita Y, Yuri T, Shikata N , Tsubura A: Curcumin suppresses $\mathrm{N}$-methyl-N- nitrosourea -induced photoreceptor apoptosis in Sprague-Dawley rats. In vivo 2013;27:583590.

17 Lee SH, Jeong E, Paik SS, Jeon JH, Jung SW, Kim HB, Kim M, Chun MH, Kim IB: Cyanidin-3-glucoside extracted from mulberry fruit can reduce $\mathrm{N}$-methyl-N-nitrosourea-induced retinal degeneration in rats. Curr Eye Res 2014;39:79-87.

18 Bowen-Forbes C, Zhang Y, Nair M: Anthocyanin content, antioxidant, anti-inflammatory and anticancer properties of blackberry and raspberry fruits. J Food Compos Anal 2010;23:554-560.

19 Andersen OM, Jordheim M: The Anthocyanins; in Andersen OM and Markham KR, Flavonoids: Chemistry and Biochemistry, Boca Raton, Taylor \& Francis Press 2006, pp 471-552.

20 Canter PH, Ernst E: Anthocyanosides of Vaccinium myrtillus (bilberry) for night vision--a systematic review of placebo-controlled trials. Surv Ophthalmol 2004;49:38-50.

21 Nakaishi H, Matsumoto H, Tominaga S, Hirayama M: Effects of black current anthocyanoside intake on dark adaptation and VDT work-induced transient refractive alteration in healthy humans. Altern Med Rev 2000;5:553-562.

22 Tanaka J, Kadekaru T, Ogawa K, Hitoe S, Shimoda H, Hara H: Maqui berry (Aristotelia chilensis) and the constituent delphinidin glycoside inhibit photoreceptor cell death induced by visible light. Food Chem 2013;139:129-137.

23 Liu Y, Song X, Han Y, Zhou F, Zhang D, Ji B, Hu J, Lv Y, Cai S, Wei Y, Gao F, Jia X: Identification of anthocyanin components of wild Chinese blueberries and amelioration of light-induced retinal damage in pigmented rabbit using whole berries. J Agric Food Chem 2011;59:356-363.

24 Jang YP, Zhou J, Nakanishi K, Sparrow JR: Anthocyanins protect against A2E photooxidation and membrane permeabilization in retinal pigment epithelial cells. Photochem Photobiol 2005;81:529-536.

25 Matsunaga N, Imai S, Inokuchi Y, Shimazawa M, Yokota S, Araki Y, Hara H: Bilberry and its main constituents have neuroprotective effects against retinal neuronal damage in vitro and in vivo. Mol Nutr Food Res 2009;53:869-877. 


\section{Cellular Physiology Cell Physiol Biochem 2016;38:893-908

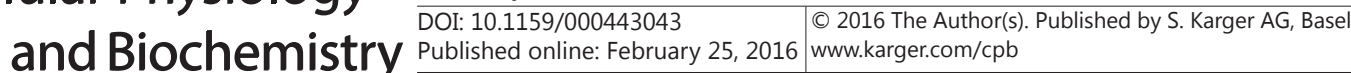 \\ Tao et al.: Retina, Neurotoxicity}

26 Paik SS, Jeong E, Jung SW, Ha TJ, Kang S, Sim S, Jeon JH, Chun MH, Kim IB: Anthocyanins from the seed coat of black soybean reduce retinal degeneration induced by N-methyl-N-nitrosourea. Exp Eye Res 2012;97:55-62.

27 Noda Y, Kaneyuki T, Mori A, Packer L: Antioxidant activities of pomegranate fruit extract and its anthocyanidins: delphinidin, cyanidin, and pelargonidin. J Agric Food Chem 2002;50:166-171.

28 Tao Y, Chen T, Liu B, Yang GQ Peng G, Zhang H, Huang YF: The neurotoxic effects of N-Methyl- N-nitrosourea on the electrophysiological property and visual signal transmission of rat's retina. Toxicol Appl Pharmacol 2015;286:44-52.

29 Tao Y, Chen T, Liu B, Xue JH, Zhang L, Xia F, Pang JJ, Zhang ZM: Visual signal pathway reorganization in the Cacna1f mutant rat model. Invest Ophthalmol Vis Sci 2013;54:1988-1997.

30 Zhong Y, Li J, Wang JJ, Chen C, Tran JT, Saadi A, Yu Q Le YZ, Mandal MN, Anderson RE, Zhang SX: X-Box binding protein 1 is essential for the anti-oxidant defense and cell survival in the retinal pigment Epithelium. Plos one 2012;7:e38616.

31 Groeger G, Mackey AM, Pettigrew CA, Bhatt L, Cotter TG: Stress-induced activation of Nox contributes to cell survival signalling via production of hydrogen peroxide. J Neurochem 2009;109:1544-1554.

32 Doonan F, Groeger G, Cotter TG: Preventing retinal apoptosis--is there a common therapeutic theme? Exp Cell Res 2012;318:1278-1284.

33 Komeima K, Usui S, Shen J, Rogers BS, Campochiaro PA: Blockade of neuronal nitric oxide synthase reduces cone cell death in a model of retinitis pigmentosa. Free Radic Biol Med 2008;45:905-912.

$34 \mathrm{Pu}$ M, Xu L, Zhang H: Visual response properties of retinal ganglion cells in the Royal College of Surgeons dystrophic rat. Invest Ophthalmol Vis Sci 2006;47:3579-3585.

35 Wu SM: From retinal circuitry to eye diseases-in memory of Henk Spekreijse. Vision Res 2009;49:992995.

36 Puchalla JL, Schneidman E, Harris RA, Berry MJ: Redundancy in the population code of the retina. Neuron 2005;46:493-504.

37 Stasheff SF: Emergence of sustained spontaneous hyperactivity and temporary preservation of OFF responses in ganglion cells of the retinal degeneration (rd1) mouse. J Neurophysiol 2008;99:1408-1421.

38 Stasheff SF, Shankar M, Andrews MP: Developmental time course distinguishes changes in spontaneous and light-evoked retinal ganglion cell activity in rd1 and rd10 mice. J Neurophysiol 2011;105:3002-3009.

39 Marc RE, Jones BW, Anderson JR, Kinard K, Marshak DW, Wilson JH, Wensel T, Lucas RJ: Neural reprogramming in retinal degeneration. Invest Ophthalmol Vis Sci 2007;48:3564-3571.

40 Dräger UC, Hubel DH: Studies of visual function and its decay in mice with hereditary retinal degeneration. J Comp Neurol 1978;180:85-114.

41 Margolis DJ, Detwiler PB: Cellular origin of spontaneous ganglion cell spike activity in animal models of retinitis pigmentosa. J Ophthalmol DOI:10.1155/2011/507037.

42 PahlbergJ, SampathAP: Visual threshold is set by linear and nonlinear mechanisms in the retina that mitigate noise: how neural circuits in the retina improve the signal-to-noise ratio of the single-photon response. Bioessays 2011;33:438-447.

43 Walle T: Absorption and metabolism of flavonoids. Free Radic Biol Med 2004;36:829-837.

44 Wang LS, Stoner GD: Anthocyanins and their role in cancer prevention. Cancer Lett 2008;269:281-290.

45 Sehitoglu MH, Farooqi AA, Qureshi MZ, Butt G, Aras A: Anthocyanins: targeting of signaling networks in cancer cells. Asian Pac J Cancer Prev 2014;15:2379-81.

46 Matsumoto H, Nakamura Y, Iida H, Ito K, Ohguro H: Comparative assessment of distribution of blackcurrant anthocyanins in rabbit and rat ocular tissues. Exp Eye Res 2006;83:348-356.

47 Kalt W, Blumberg JB, McDonald JE, Vinqvist-Tymchuk MR, Fillmore SA, Graf BA, O'Leary JM, Milbury PE: Identification of anthocyanins in the liver, eye, and brain of blueberry-fed pigs. J Agric Food Chem 2008;56:705-712.

48 Bartikova H, Skalova L, Drsata J, Bousova I: Interaction of anthocyanins with drug-metabolizing and antioxidant enzymes. Curr Med Chem 2013;20:4665-4679.

49 Rodriguez Fragoso L, Martinez-Arismendi JL, Orozco-Bustos D, Reyes-Esparza J, Torres E, Burchiel SW: Potential risks resulting from fruit/vegetable-drug interactions: effects on drug-metabolizing enzymes and drug transporters. J Food Sci 2011;76:112-124. 